thebmj

Practice Lesson of the Week

\title{
Extensive transmission of Mycobacterium tuberculosis from 9 year old child with pulmonary tuberculosis and negative sputum smear
}

BMJ 2008; 337 doi: http://dx.doi.org/10.1136/bmj.a1184 (Published 28 August 2008) Cite this as: BMJ 2008;337:a1184

S Paranjothy, Walport clinical lecturer in public health medicine ${ }^{1}$, M Eisenhut, consultant paediatrician2, M Lilley, consultant in health protection 3 , $\mathbf{S}$ Bracebridge, regional epidemiologist 4 , I Abubakar, consultant epidemiologist 5 , head of tuberculosis section $6, \mathbf{R}$ Mulla, consultant microbiologist 2 , K Lack, senior health protection nurse (TB) 3 , D Chalkley, lead TB nurse 7 , J Howard, specialist registrar4, S Thomas, specialist registrar4, M McEvoy, unit director 3

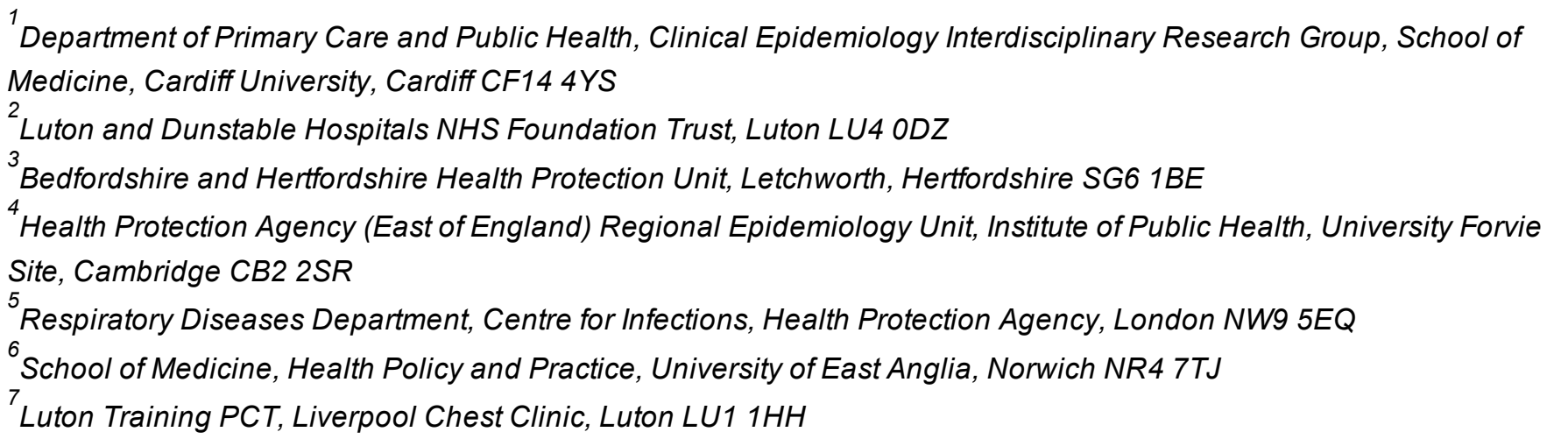

Correspondence to: S Paranjothy paranjothys@cf.ac.uk

- Accepted 9 July 2008

A negative sputum smear does not exclude substantial risk of infection from patients with pulmonary tuberculosis

Patients with pulmonary tuberculosis and either a positive sputum smear or cavitating pulmonary lesions have been considered to be infectious1 as these indicate higher bacterial load accumulating with longer duration of infection. The source of infection in outbreaks among children is usually an adult with these features. 2 Children have been considered less likely to transmit infection because they were unlikely to expectorate infective droplet nuclei. In the absence of a positive sputum smear, guidelines do not recommend screening of wider contacts in addition to household contacts, 3 although in the United States, contact screening is recommended if resources are sufficient. 4 Here, we report extensive transmission of tuberculosis in a junior school in Luton, England, from a child with pulmonary tuberculosis in whom a sputum smear was negative. 


\section{The index case}

The index case was a 9 year old boy of Black African ethnicity born in the United Kingdom. He was investigated by his family doctor because of a six year history of recurrent cough evolving into a daily chronic cough with night sweats and weight loss for eight weeks before referral to a paediatrician. The cough was occasionally productive with one episode of haemoptysis. He continued to attend school while symptomatic. A chest $x$ ray film showed a right upper lobe consolidation and multiple poorly defined opacities in the right lower lobe. (figure $\underline{\Downarrow}$ ). Three sputum collections were smear negative, and culture showed Mycobacterium tuberculosis, fully sensitive to all drugs tested (isoniazid, rifampicin, pyrazinamide, ethambutol). He started chemotherapy with a two month course of pyrazinamide, rifampicin, and isoniazid, followed by four months of isoniazid and rifampicin. All household contacts of the child were screened for $M$ tuberculosis infection.

Figure1

Fig 1 First chest radiograph of the index case, a 9 year old boy with chronic cough and $M$ tuberculosis grown from sputum samples

The boy's three siblings had positive Mantoux readings, positive T-SPOT tests (Oxford Immunotec), and normal chest $x$ ray examinations. The T-SPOT test is one of two immunological tests that measure the release of interferon $\mathrm{y}$ by $\mathrm{T}$ cells in response to $M$ tuberculosis specific antigens and are commercially available in the United Kingdom. These tests correlate better with exposure to $M$ tuberculosis than the Mantoux test.3

The three siblings started chemoprophylaxis (three month course of rifampicin and isoniazid). Three children from further extended family had positive T-SPOT tests; two of these had normal chest radiographs and started chemoprophylaxis. The third, asymptomatic child had bilateral hilar lymphadenopathy on chest radiography and started chemotherapy. All five adults in the immediate and extended family screened positive and required chemoprophylaxis. In the absence of an apparent adult source of infection among household contacts, screening was extended to include the index case's school contacts.

\section{School contacts of the index case}

The boy who was the index case attended a junior school with 200 other pupils aged 8 to 12 years. Initially, all contacts within his year group (classes 5 and 6 ) who shared lessons and class teachers were screened using Mantoux tests. In his year group 36\% of pupils were white; $23 \%$ were Bangladeshi, Indian, or Pakistani; $14 \%$ were Black African or Black Caribbean; and $21 \%$ were of mixed ethnicity (white and either Asian or Black Caribbean parents). Positive Mantoux tests were confirmed using T-SPOT tests, in accordance with national guidance.3

As the infection rate among these contacts was high $(30 / 43=69.7 \%)$, screening was extended to include all pupils. Owing to the numbers involved and because the school term was coming to an end, pupils were screened using the T-SPOT test and chest radiography. Each chest radiograph was read by a paediatrician with expertise in tuberculosis and independently read again and reported by a consultant radiologist. Children with pulmonary parenchymal changes on chest radiography were regarded as potentially infectious and investigated by obtaining three sputum and three gastric lavage samples. As we were seeking an adult source of infection, adults with at least eight hours' cumulative contact with 
the school (including staff, support workers, and adult visitors) during the previous eight months were invited for chest radiography.

Altogether, 85 children (42\% of all school pupils) had positive T-SPOT tests; of these, 16 had hilar lymphadenopathy but were asymptomatic and two had pulmonary parenchymal changes and were admitted for gastric lavage and sputum collection. One of these two, an 8 year old boy in year 3 , had acid fast bacilli on gastric lavage and a negative sputum smear (second case).

All adult chest $x$ ray examinations were normal, and no additional cases of active or latent tuberculosis were detected among the household contacts of the children diagnosed with active tuberculosis on screening.

\section{Epidemiological findings}

Infection rates were highest in the class of the index case (79\%). The infection rate in this class was significantly higher than that among the other pupils at the school $(35 \%)(P<0.01)$. Infection rates did not correlate with ethnicity.

\section{Microbiological investigations and DNA fingerprinting}

DNA fingerprinting-using a 15 loci based typing scheme known as mycobacterium interspersed repetitive units variable number tandem repeats (MIRU-VNTR) - showed that the $M$ tuberculosis strain (42433 $2331514321, \mathrm{~S}$ lineage) of the index case was genetically identical to a strain isolated six years previously in an adult from the extended family of the index case who was living in London at the time of his diagnosis but had since returned to Africa. $M$ tuberculosis grown from the gastric lavage sample of the second case detected in this outbreak was found to be genetically indistinguishable from the sample of the index case.

\section{Discussion}

Smear negative pulmonary tuberculosis is significantly less infectious than smear positive disease, and although transmission has been documented, 5 this is the first report of extensive transmission from a child.

The decision to screen wider contacts of the index case was not consistent with current national guidelines 3 but was driven by the high rate of infection among the children who were household contacts of the index case. We were unaware then of the extended family contact who had previously had a diagnosis of tuberculosis and had no direct link with the school. We screened staff and pupils attending the school as we were seeking an adult source of infection and any other infected children.

Our results led us to hypothesise that the index case was the source case for the infected school children. Several findings supported this hypothesis. Firstly, the infection rate in the class of the index case was higher than the rates in the other classes. Secondly, the identification of an epidemiological and DNA fingerprinting link of the index case with an adult contact who had had no contact with the school of the index case supports the hypothesis that the index case was the source case of the school outbreak. The shared strain type between the two children at the junior school provided good evidence that child to child transmission occurred.

Thirdly, the strain identified is relatively rare in England as it was found in only two other patients in the 
national strain typing database that currently holds 17305 records. Both of these were from the same West African country as the index case but had no epidemiological link with the index case or the school.

Finally, the area of Luton where the school is located has an annual incidence of tuberculosis of 45 per 100000 people, meeting the definition of a high incidence area.3 Previous screening of school contacts in this area following cases of sputum smear positive pulmonary tuberculosis in teenage children in the past two years found rates of positive Mantoux tests of pupils between $0 \%$ and $2.4 \%$. This indicated that most of the high rate of infection in the school (42\%) could not be explained by the expected rate of infection associated with the prevalence in the area. The high rate of infection among school children may have been facilitated by poor ventilation and all pupils sharing a room for lunch.

Our report, together with evidence from other studies,4 678 highlights the need for increased awareness of pulmonary tuberculosis as a differential diagnosis in children who present to general practitioners with a long history of recurrent or chronic cough.

\section{Conclusion}

This report is evidence of extensive transmission of $M$ tuberculosis from a young child with smear negative tuberculosis. National guidelines, which place emphasis on sputum smear positivity as main indicator of infectivity for starting contact tracing outside the household setting, 3 may have to be revised, with longstanding exposure to a coughing tuberculous patient with extensive pulmonary lesions included in the criteria for contact screening.

\section{Notes}

Cite this as: $B M J 2008 ; 337: a 1184$

\section{Footnotes}

- Contributors: All authors were involved in the outbreak investigation. SP was responsible for data analysis and the first draft of this paper. All authors contributed to interpreting the data, critical revisions, and approving the final version to be published. MMcE is the guarantor for this article.

- Funding: No special funding.

- Competing interests: None declared.

- Provenance and peer review: Not commissioned; externally peer reviewed.

- Patient consent obtained.

\section{References}

1. Hertzberg G. The infectiousness of human tuberculosis; an epidemiological investigation. Acta Tuberc Scand Supp/1957;38:1-147.

2. Singh M, Mynak M L, Kumar L, Matthew JL, Jindal SK. Prevalence and risk factors for transmission of infection among children in household contact with adults having pulmonary tuberculosis. Arch Dis Child2005;90:624-8.

3. National Collaborating Centre for Chronic Conditions. Tuberculosis: clinical diagnosis and management of 

tuberculosis, and measures for its prevention and control. London: Royal College of Physicians, 2006.

4. Guidelines for the Investigation of Contacts of Persons with Infectious Tuberculosis. Recommendations from the National Tuberculosis Controllers Association and Centers for Disease Control and Prevention. Morbidity and Mortality Weekly Report2005;54(RR15):1-37.

5. Behr MA, Warren SA, Salamon H, Hopewell PC, Poce de Leon A, Daley CL, et al. Transmission of Mycobacterium tuberculosis from patients smear-negative for acid-fast bacilli. Lancet 1999;353:444-9.

6. Curtis AB, Ridzon R, Vogel R, Mc Donough S, Hargreaves J, Ferry J, et al. Extensive transmission of Mycobacterium tuberculosis from a child. N Engl J Med1999;341:1491-5.

7. Loudon RG, Spohn SK. Cough frequency and infectivity in patients with pulmonary tuberculosis. Am Rev Resp Dis 1969;99:109-11.

8. Lienhardt C, Sillah J, Fielding K, Donkor S, Manneh K, Warndorff D, et al. Risk factors for tuberculosis infection in children in contact with infectious tuberculosis cases in The Gambia, West Africa.

Pediatrics2003;111:608-14. 